\title{
TÄTIGKEITSBERICHT DER INTERNATIONALEN KOMMISSION FÜR BIENENBOTANIK DER I.U.B.S. FÜR DIE JAHRE 1966-1969
}

\author{
Anna MAURIZIO und Jean LOUVEAUX \\ 3097 Liebefeld Bern, Rosenweg 9 (Suisse) \\ Station de Recherches sur l'Abeille et les Insectes sociaux, \\ 91 - Bures-sur-Yvette \\ Institut national de la Recherche agronomique
}

Der vorliegende Bericht umfasst die Tätigkeit der Internationalen Kommission für Bienenbotanik der I.U.B.S. und ihrer Arbeitsgruppen während der vier vergangenen Jahre, d.h. die Zeitspanne zwischen den Mitgliederversammlungen in Bukarest (1965) und in München (1969).

Da verhältnismässig wenige Mitglieder am XXI. Internationalen Bienenzüchterkongress in Maryland (U.S.A.) teilnehmen konnten, wurde davon abgesehen dort eine Mitgliederversammlung der Kommission anzusetzen. Nur die Arbeitsgruppe für Bestäubungsfragen hielt eine Sitzung ab.

Die Internationale Kommission für Bienenbotanik der I.U.B.S. zählt heute $\mathbf{1 7 5}$ Mitglieder in 34 Ländern. Während der Berichtsperiode verlor sie fünf Mitglieder durch den Tod (N. M. GLusHkov, U.d.S.S.R.; J. I. Hambleton, U.S.A.; B. Hazslinsky, Ungarn; W. LüdI, Schweiz; E. WohlGEMuth, Deutschland). Freiwillig sind aus der Kommission ausgetreten 17, neu eingetreten 27 Mitglieder. Neu vertreten in der Kommission sind 3 Länder (Angola, Argentinien, Belgien).

An der am 31. Juli 1969 in München abgehaltenen allgemeinen Versammlung der Kommission nahmen 24. Mitglieder aus 15 Ländern teil. Es wurden verschiedene administrative Geschäfte behandelt, darunter ein Vorschlag zur Reorganisation der Kommission. Es war der Wunsch geäussert worden, die bisher sehr lockere Organisation der Kommission etwas straffer zu gestalten. Das Präsidium legte deshalb der Mitgliederversammlung einen Reorganisationsentwurf vor. Nach eingehender Diskussion wurden folgende Beschlüsse gefasst :

$a$. J. Louveaux wird beauftragt einen Entwurf für Statuten der Kommission für Bienenbotanik auszuarbeiten und ihn mit dem Generalsekretariat der I.U.B.S. und einem beratenden Komitee zu bereinigen. Der Entwurf wird sodann den Mitgliedern schriftlich zugestellt und an der nächsten allgemeinen Versammlung zur Diskussion und Genehmigung vorgelegt.

b. Das Präsidium wird erweitert, d.h. G. VoRwoHL als Kassier gewählt.

c. Es wird ein beratendes Komitee gewählt.

d. Zur Deckung der kleinen Ausgaben der Kommission wird auf Neujahr 1970 ein Mitgliederbeitrag eingeführt. Der Beitrag soll vorläufig nur von den der Kommission angehörenden Instituten erhoben werden; er beträgt 10 Dollar jährlich.

e. Als weiteres Traktandum lag der schon 1965 von E. V. SANDulEac angemeldete Vorschlag zur Gründung einer neuen Arbeitsgruppe für "Biophysik und Genetik" vor. Es wurde beschlossen diesen Vorschlag nach abgeschlossener Reorganisation der Kommission nochmals in Erwägung zu ziehen. 
Die Internationale Kommission für Bienenbotanik veranstaltete während der Berichtsperiode folgende Tagungen und gab folgende Publikationen heraus oder war an ihnen beteiligt :

\section{Tagungen :}

- Teilnahme an der 2. Internationalen Konferenz für Palynologie in Utrecht 1966.

- Arbeitstagung der Gruppe für Honigforschung in Stuttgart-Hohenheim 1967.

- Mitarbeit der Arbeitsgruppe für Honigforschung im Methodenkomitee des Codex Alimentarius Europaeus; Teilnahme an den Sitzungen des Komitees in Berlin 1967, 1968.

- Teilnahme an der konstituierenden Versammlung der Association des Palynologues de langue française in Paris 1968. 1969.

- Skandinavische Arbeitstagung für Fragen der Bestäubung und des Bienenschutzes in Asker

- Gemeinsame Sitzung der Arbeitsgemeinschaft der Institute für Bienenforschung und der Kommission für Bienęnbotanik, Arbeitsgruppe für Honigtauforschung, am wissenschaftlichen Symposium des XXII. Internationalen Bienenzüchterkongresses in München 1969.

\section{Publikationen :}

- Bericht über die Tätigkeit der Internationalen Kommission für Bienenbotanik der I.U.B.S. in den Jahren 1962-1965 (Bee World 46 : 132, 1965; Ann. Abeille 8 : 325, 1965; Zeitschr. f. Bienenforschg. $8: 267,1966$ ).

- Proceedings of the 2nd International Symposium on Pollination (Akerberg/Crane, Bee Res. Assoc., Suppl. Bee World, Vol. 47, 1966).

Paris 1965).

- Broschüre "Pollens de Plantes mellifères d'Europe" (Maurizio/Louveaux, U.G.A.F.,

- Sonderheft über Heidehonig (Ann. Abeille 9 [4], 1966).

- Sonderheft über die Tagung für Fragen der Honigherkunftsbestimmung in StuttgartHohenheim, 1967 (Zeitschr. f. Bienenforschg. 9 [5], 1968).

- Microscopic examination of honey (Joint FAO/WHO Food Standard Programme, Codex Comitee on Methods of Analyses and Sampling, Codex/Analys/68/10, 1968).

— Zirkulare No. 22-26 der Internationalen Kommission für Bienenbotanik der I.U.B.S. 19651969.

- Listen No. 7 und 8 neuer Literatur über Honig- und Pollenforschung, 1965, 1969.

- Mitarbeit am Traité de Biologie de l'Abeille (Chauvin, Masson, Paris 1968).

- Mitarbeit am "Waldhonigbuch" (Kloft/Maurizio/KaEser, Ehrenwirth, München 1965).

- Mitarbeit am "Trachtpflanzenbuch" (Maurizio/Grafl, Ehrenwirth, München 1969).

Die Tätigkeit der Kommission für Bienenbotanik spielt sich vor allem in den Arbeitsgruppen ab. Besonders lebhaft gestaltete sich die Arbeit in den Gruppen für Honigforschung, Honigtauforschung und Bestäubungsfragen. Die Arbeitsgruppen für Bienenschutz und für Mediterranflora befinden sich in Reorganisation.

\section{BERICHTE ÜBER DIE TÄTIGKEIT DER ARBEITSGRUPPEN}

\section{1. - Arbeitsgruppe für Honigforschung}

(Berichterstatter J. Louveaux, A. Maurizio)

Als wichtigste Tätigkeit der Arbeitsgruppe für Honigforschung während der Jahre 1966-1969 kann die Tagung betrachtet werden, die vom 11.-13. Mai 1967 in Stuttgart-Hohenheim stattfand. Sie vereinigte etwa 30 Teilnehmer aus 9 Ländern, darunter neben Spezialisten für Honigmikroskopie auch mehrere Chemiker. So wurde erstmals das seit mehreren Jahren verfolgte Ziel erreicht, die Arbeiten der Gruppe auf das ganze Gebiet der Honigforschung auszudehnen. An der Tagung wurden 14. Referate gehalten, die in einem Sonderheft der Zeitschrift für Bienenforschung veröffentlicht sind (Bd. 9, Heft 5, 1968). Die Mehrzahl der Referate befasste sich mit Fragen der Pollenanalyse des Honigs, es befinden sich darunter aber auch mehrere rein chemische Arbeiten, sowie eine Zusammenfassung über die Methoden zur Charakteristik von Sortenhonigen. Im Anschluss an ein Referat über die Grundzüge einer modernen Pollenbeschreibung im Rahmen der Bienen- und Honigkunde fand eine ausgiebige Diskussion statt. 
Die Arbeitsgruppe war auch vertreten an der 2. internationalen Konferenz für Palynologie in Utrecht 1966 und an der konstituierenden Versammlung der Association des Palynologues de langue française in Paris 1968. An der Konferenz in Utrecht wurden zwei Referate über Melisso. palynologie gehalten, die vor allem den Zweck hatten das Interesse unserer Kommission an der Palynologie im allgemeinen zu unterstreichen. Wenn aulch die Methodik der Melissopalynologie in mancher Hinsicht von derjenigen der allgemeinen Paynologie abweicht, was eine weitgehende Spezialisation mit sich bringt, müssen die Honig-Spezialisten doch engen Kontakt mit den Palynologen pflegen. Das Bedürfnis nach engeren Kontakten hat mehrere unter uns veranlasst der Association des Palynologues de langue française beizutreten. Der Wunsch nach engeren Kontakten ist übrigens gegenseitig, was seinen Ausdruck fand in der Aufnahme eines Mitgliedes der Arbeitsgruppe in das neu gegrïndete internationale Komitee für Palynologie.

Das seit langem angestrebte Ziel einer Zusammenfassung und internationalen Bereinigung der Methodik der Melissopalynologie in einer einzigen, jedermann leicht zugänglichen Publikation konnte während der Berichtsperiode verwirklicht werden. Die in deutscher, französischer und englischer Sprache veröffentlichte "Methodik der Melissopalynologie " tritt an die Stelle der 1963 durch die Kommission für Bienenbotanik herausgegebenen "Methoden der Honigpollenanalyse". Die neue Publikation ist um vieles vollständiger, sie umfasst nicht nur die Untersuchungsmethodik, sondern legt auch in klarer Weise die Terminologie fest und gibt genaue Hinweise auf die Art der Auswertung der Untersuchungsergebnisse. Sie dient als Grundlage für die Aufnahme der Melissopalynologie in den Methoden des Codex Alimentarius Europaeus und ist dem Methodenkomitee des Codex zur Diskussion vorgelegt worden (Codex/Analys/68/10, 1968). Eine solche Neufassung der Methodik war wichtig, um der Honigpollenanalyse eine Form zu verleihen, die geeignet ist auf internationaler Ebene, und besonders im Rahmen des Codex Alimentarius anerkannt zu werden.

Zusammenfassend kann gesagt werden, daß die Honigforschung, die vor einigen Jahren an Interesse zu verlieren schien, heute wieder ihre volle Bedeutung zurückerlangt hat. Die Ursachen dieser Neubelebung sind ohne Zweifel in den heutigen wirtschaftlichen Verhältnissen zu suchen. Die Produzenten, der Handel und die Verbraucher verlangen immer mehr, daß die Lebensmittel, darunter der Honig, exakten Qualitätsnormen entsprechen und eine sichere Garantie der Herkunft bieten. Die Fortschritte der Analysemethoden geben heute den offiziellen, mit der Lebensmittelkontrolle betrauten Amtsstellen neue Möglichkeiten zur Feststellung der Qualität, Herkunft und Echtheit der Produkte. Die Arbeitsgruppe für Honigforschung der internationalen Kommission für Bienenbotanik zählt es zu ihren Aufgaben, auf internationaler Ebene zum Fortschritt und zur Verbreitung wissenschaftlicher und technischer Kenntnisse über die Zusammensetzung und Analyse des Honigs beizutragen. Diesem Zweck dienen unter anderm die in regelmässigen Abständen herausgegebenen Listen neuer Literatur über Honig- und Pollenforschung.

\section{2. - Arbeitsgruppe für Nektarforschung}

(Berichterstatter R. W. Shuet, G. F. Townsend)

Die Tätigkeit der Arbeitsgruppe für Nektarforschung beruht zur Hauptsache auf einem Austausch von Informationen und Literaturhinweisen und auf Vorschlägen für weitere Untersuchungen. Ein Bericht über neue, in Frankreich, Deutschland, Israel, Polen, Grossbritannien, den Vereinigten Staaten und Kanada ausgeführten Arbeiten und über zukünftige Aufgaben der Nektarforschung wird demmächst in der Zeitschrift Bee World erscheinen.

Vom Standpunkt der praktischen Anwendbarkeit verfolgen Untersuchungen über Nektar vor allem zwei Probleme : die Verbesserung der Honigproduktion und die Steigerung der Pflanzenerträge durch bessere Bestäubung. Zur Lösung dieser Probleme können mehrere Methoden beigezogen werden :

a. Beeinflussung der Nektarspender durch Züchtung oder durch Aenderung der Umweltsbedingungen.

b. Selektion der Arten, die sich für die einzelnen Gebiete besonders eignen.

c. Selektion der bestäubenden Insekten. Alle drei Methoden stützen sich auf exakte Grundlagenforschung. Folgende Fragen stehen heute im Vordergrund des Interesses :

1. Einfluss der Temperatur : Bei gewissen Pflanzenarten besteht ein deutlicher Schwellenwert der Temperatur, unterhalb dessen keine Nektarsekretion stattfindet. Das lässt eine Abhängigkeit der Nektarsekretion von einem oder mehreren Fermentsystemen vermuten. Frage : welche Fermentsysteme der Pflanze sind für die Nektarsekretion wichtig? 
2. Voraussage der Nektartracht: In Gebieten mit nektarspendenden Wäldern bestehen grosse, bisher nicht abgeklärte Schwankungen der Nektartracht von Jahr zu Jahr. Frage : wird die Nektartracht durch die vorhergehende Saison beeinflusst? Kann die Nektartracht vorausgesagt werden? Vorhersagen der zu erwartenden Nektartracht wären von grosser Bedeutung für die Wanderbienenzucht gewisser Länder (z. B. Australien).

3. Anlockung von Bestäubern : Frage : worauf beruht die Attraktivität der Pflanzen für Insekten? G. R. WyKes fand, dass das gegenseitige Mengenverhältnis der Hauptzucker im Nektar einen gewissen Einfluss hat auf die von der Honigbiene getroffene Auswahl der Futterquellen. Spielen Aromastoffe in dieser Hinsicht eine Rolle? Die Untersuchung von duftenden und duftlosen Varientäten der gleichen Pflanzenart könnten hier Hinweise geben.

4. Hormonale Steuerung : Frage : wieweit sind hormonate Vorgänge an der Nektarsekretion beteiligt und wie wird die Nektarsekretion mit der Blütenentwicklung, der Pollenreifung und der Narbenempfänglichkeit koordiniert?

\section{3. - Arbeitsgruppe für Honigtauforschung}

(Berichterstatter W. KLoft)

In die Berichtsperiode fällt die Veröffentlichung wichtiger Buchpublikationen durch Mitglieder der Arbeitsgruppe. Durch Zusammenarbeit von W. Kaeser, A. Fossel, J. Schels, A. Maurizio und W. KLoFT ist das "Wcldhonigbuch" (Ehrenwirth Verlag 1965) entstanden, welches den Versuch macht, das gegenwärtige Wissen zusammenzufassen und zugleich die Lücken aufzuzeigen. Diese Publikation wird ergänzt und erweitert durch eine Buchveröffentlichung von Haragsim (1966) sowie ein Kapitel von Müller in "Die Bienenweide "(Ulmer Verlag 1967). Das Traité de Biologie de l'Abeille (Masson 1968) hat mit Beiträgen von Maurizıo und KLofT diese Befunde im französischen Sprachbereich weiterverbreitet. Besprechungen der genannten Publikationen auch im englischen und russischen Schrifttum zeigen das grosse Interesse an Honigtau-Fragen. Im ganzen lässt sich feststellen, dass 1) durch zunehmendes Wissen um die Zusammenhänge in immer weiter. gehenderem Masse die Bedeutung der Honigtautrachten in Mittel-, West-, Süd-, Südost- und Osteuropa, darüber hinaus aber auch in Asien, Neusseeland und Amerika erkannt wird. 2. Die zunehmende Herbizid- und Insektizid-Anwendung in der hochentwickelten Landwirtschaft verweist immer stärker auf Honigtautrachten zur Existenzicherung der Imkerei. 3. Zunehmende Verbreitung der Kenntnisse von Honigtau-Erzeugern und Tracht fördert in Zusammenarbeit zwischen Praxis, Bienenwissenschaft und Forstwirtschaft die Erschliessung der Trachtquellen. Prognosedienste, z. T. mit Unterstützung der öffentlichen Hand und der Rundfunkanstalten sind im Entstehen resp. haben sich bereits bewährt.

Bei all' diesen Massnahmen waren kollegiale Kontakte und vielfältige Zusammenarbeit zwischen den Mitgliedern der Arbeitsgruppe entscheidende Voraussetzung für bereits erzielte Erfolge. Sie werden gefördert durch Korrespondenzen, Teamarbeit und persönliche Treffen auf Fachtagungen und Kongressen - - sehr wichtig war der XIII. Internationale Entomologenkongress Moskau 1968. Ein für den Herbst 1968 in Halle vorgesehenes Spezialistentreffen, bei dem Experten aus BRD, CSR, DDR, Holland und Polen zusammentreffen sollten, konnte leider nicht stattfinden. Hauptanliegen bleibt weiterhin die Klärung systematisch-taxonomischer Probleme bei Lachniden, vor allem der an Pinus saugenden Arten. Mit diesem Komplex wird sich die Arbeitsgruppe auch in den nächsten Jahren auseinandersetzen müssen.

Im übrigen wird auf das in München stattfindende Symposium " Honigtau und Honigtauerzeuger " verwiesen, welches mit 11 Referaten und einem Film, vorgetragen von Mitgliedern aus 7 Ländern die gegenwärtig bearbeiteten Probleme und damit auch die Aktivität der Arbeitsgruppe widerspiegeit.

\section{4. - Arbeitsgruppe für Bienenschutz}

(Berichterstatter K. STUTE)

Die Arbeiten in dieser Gruppe erstreckten sich auf zwei Gebiete :

1. Prüfung von Pflanzenschutzmitteln auf ihre Wirkung gegenüber Bienen.

2. Nachweis derartiger Stoffe in toten Bienen. 
1. Innerhalb des Gebietes der Bundesrepublik Deutschland wird an den einzelnen Prüfstetlen (10) nach einheitlichen, anerkannten Richtlinjen gearbeitet, die im Jahre 1969 veröffentlicht wurden. Es werden hierin Anleitungen für die Bestimmung der Atem-, Kontakt- und Frassgiftwirkung im Laboratorium, für die Anlage von Zelt- und Freilandversuchen und für die Zusammenstellung der Versuchsergebnisse gegeben. In den wesentlichen Punkten decken sich diese Methoden mit denen, die bereits vor Jahren auch innerhalb der internationalen Arbeitsgruppe zur Anwendung empfohlen wurden. Es hat sich in Deutschland herausgestellt, dass die Ergebnisse der einzelnen Prüfstellen für die jeweiligen Präparate innerhałb der bei biologischen Versuchen zu erwartenden Abweichungen so gut übereinstimmen, dass es bisher in den meisten Fällen möglich war, innerhalb einer Prüfsaison (Mai bis September) eine Anerkennung über die Fingruppierung der betreffenden Stoffe auszusprechen.

In der Bundesrepublik Deutschland steht man nach wie vor auf dem Standpunkt, eine Zweiteilung der Pflanzenschutzmittel in "bienengefährliche " und " bienenungefährliche " zu belassen. Gestützt wird diese Einteilung durch die Tatsache, dass im Bundesgebiet sehr verschiedene Pflanzenkulturen auf teilweise kleinen Flächen angebaut werden, wobei die Ueberwachung der notwendigen Bekämpfungsaktionen durch amtliche Stellen auf erhebliche Schwierigkeiten stösst. Es ist anderseits bekannt, dass einige Länder drei und mehr Gruppen hinsichtlich ihrer Wirkung auf Bienen unterscheiden. Da hierfür meistens die örtlichen Verhältnisse ausschlaggebend sind, wird es kaum möglich sein, auf diesem Gebiet eine international einheitliche Gruppierung zu treffen.

Die Anregung, einheitliche Bezeichnungen für die Pflanzenschutzmittel in den verschiedenen Ländern einzuführen, wird sehr begrüsst und weiter verfolgt. Im übrigen werden die Mitglieder dieser Arbeitsgruppe in Zukunft eine Zusammenstellung der in Deutschland als bienenungefährlich anerkannten Pflanzenschutzmittel erhalten. Es wird vorgeschlagen, ähnliche Mitteilungen anderẹ Länder dem Berichterstatter zuzuleiten, damit eine Koordinierung möglich ist.

2. Auf dem Sektor des Nachweises von Pflanzenschutzmitteln in toten Bienen hat es in den einzelnen Ländern, wo derartige Fragen akut sind, nicht gefehlt. Es werden die verschiedensten Methoden angegeben. Der Berichterstatter würde es dankbar begrüssen, wenn die jeweiligen Verfahren in kurzgefasster Form mitgeteilt werden könnten, um den Mitgliedern der Arbeitsgruppe eine ähnliche Zusammenstellung geben zu können, wie es im Jahre 1960 erfolgte.

Von mehreren Seiten erfolgt die Anregung, die nebeneinander bestehenden Arbeitsgruppen auf diesem Sektor zusammenzuschliessen. Es sei hier die Bemerkung erlaubt, dass die Arbeitsgruppe für Bienenschutz der internationalen Kommission für Bienenbotanik seit vielen Jahren besteht und entscheidend zu einer internationalen Zusammenarbeit auf dem Sektor "Bienenschutz " beigetragen hat. Um diese Zusammenarbeit zu beleben ergeht hierdurch die Einladung an alle Interessenten, sich im Jahre 1971 in Celle (Deutschland) zusammenzufinden.

\section{5. - Arbeitsgruppe für Bestäubungsfragen}

(Berichterstatter E. AKerberg, G. Julén, N. Holm)

Während der Berichtsperiode ist der Bericht über das Symposium für Bestäubung in London 1964 erschienen (Proceedings of the 2nd International Symposium on Pollination, Bee Res. Association 1966).

In Verbindung mit dem XXI. Internationalen Bienenzüchterkongress in Maryland 1967 fand eine Tagung für Bestäubungsfragen statt, an welcher 25 Spezialisten teilnahmen. Es wurden folgende Fragen diskutiert :

1. Beiziehung der Apicultural Abstracts für Besprechungen von Arbeiten über Bestäubung.

2. Organisation des nächsten Symposiums für Bestäubung. Die Mehrzahl der Teilnehmer vertrat die Ansicht, dass ein solches Symposium mit dem XXII. Internationalen Bienenzüchterkongress in München 1969 verbunden werden sollte.

3. Diskussion über Insektenbestäubung verschiedener Kulturpflanzen.

Es bestand demnach die Absicht ein Symposium für Bestäubungsfragen in Verbindung mit dem Bienenzüchterkongress in München zu veranstalten. Eine diesbezügliche Anfrage beim Kongress-Sekretariat wurde leider so spät beantwortet, dass die Arbeistgruppe es nicht für ratsam hielt in so kurzer Zeit ein Symposium zu organisieren. 
Ein Nordisches Symposium fand am 22./23. Juli 1969 in Asker, Norwegen statt. Unter andern war daran A. MAURIzıo anwesend. Eine Kurzfassung der an diesem Symposium gehaltenen Vorträge wird demnächst in der Zeitschrift Bee World erscheinen. Während der Tagung fand eine Besprechung im kleinen, hauptsächlich aus Vertretern der Nordischen Länder bestehenden Kreise statt. Es wurde daran beschlossen weiterhin in engem Kontakt mit der Internationalen Kommission für Bienenbotanik zu arbeiten und diese Frage am nächsten Symposium zur Diskussion vorzulegen. Weiter wurde der Wunsch geäussert, die Apicultural Abstracts auszubauen und auf Arbeiten aus dem Gebiete der Bestäubung zu erweitern.

Die Arbeitsgruppe erhielt eine Einladung aus Australien mit dem Vorschlag 1972, in Verbindung mit dem Entomologen-Kongress ein Symposium für Bestäubungsfragen zu organisieren. Vieles spricht für die Abhaltung eines solchen Symposiums in Australien. Näheres darüber wird in der Zeitschrift Bee World veröffentlicht.

\section{6. - Arbeitsgruppe für Mediterranflora}

(Berichterstatter J. LiNDER, J. RiHAR)

Die reorganisierte Gruppe für Mediterranflora legt ein mehrere Jahre umfassendes Arbeitsprogramm vor. Die Arbeiten sollen in den einzelnen Ländern begonnen und dann auf das ganze Mediterranbecken ausgedehnt werden, wobei mehrere Laboratorien in Mittcleuropa ihre Mitarbeit zugesagt haben. Zweck der Arbeiten ist, die Lebens- und Trachtverhältnisse der Bienen im Mediterrangebiet näher zu untersuchen.

Die geplanten Untersuchungen umfassen zunächst folgende Fragen :

1. Nektarien und Nektartracht von verschiedenen Pflanzenarten, mit Berücksichtigung der Wüstenpflanzen.

2. Pollendarbietung und Pollentracht von verschiedenen Pflanzenarten, mit Berücksichtigung der Wüstenpflanzen.

3. Mikroskopische Untersuchungen an Honigen aus verschiedenen Gegenden des Mediterrangebietes und Beschreibung der dort geernteten Honigtypen.

Es ist vorgesehen im Verlaufe der nächsten Jahre eine Arbeitstagung der Gruppe für Mediterran. flora einzuberufen. 